\title{
BMJ Open Sex differences in auditory verbal hallucinations in early, middle and late adolescence: results from a survey of 17 451 Japanese students aged 12-18 years
}

\author{
Yoko Morokuma, ${ }^{1}$ Kaori Endo, ${ }^{2}$ Atushi Nishida, ${ }^{2}$ Syudo Yamasaki, ${ }^{2}$ \\ Shuntaro Ando, ${ }^{2,3}$ Yuko Morimoto, ${ }^{2}$ Miharu Nakanishi, ${ }^{2}$ Yuji Okazaki, ${ }^{4}$ \\ Toshi A Furukawa, ${ }^{5}$ Shigeru Morinobu, ${ }^{1}$ Shinji Shimodera ${ }^{1}$
}

To cite: Morokuma Y, Endo K, Nishida A, et al. Sex differences in auditory verbal hallucinations in early, middle and late adolescence: results from a survey of 17 451 Japanese students aged 12-18 years. BMJ Open 2017;7:e015239. doi:10.1136/ bmjopen-2016-015239

- Prepublication history and additional material for this paper are available online. To view these files please visit the journal online (http://dx.doi. org/10.1136/bmjopen-2016015239).

YM and KE contributed equally.

Received 21 November 2016 Revised 22 February 2017 Accepted 5 April 2017

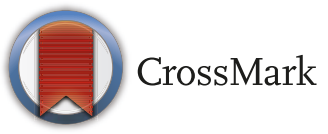

${ }^{1}$ Department of Neuropsychiatry, Kochi Medical School, Kochi University, Kochi, Japan

${ }^{2}$ Department of Psychiatry and Behavioral Science, Tokyo Metropolitan Institute of Medical Science, Tokyo, Japan

${ }^{3}$ Department of Neuropsychiatry, Graduate School of Medicine,

The University of Tokyo, Tokyo, Japan

${ }^{4}$ Kouseikai Michinoo Hospital, Nagasaki, Japan

${ }^{5}$ Department of Health

Promotion and Human Behavior, Graduate School of Medicine and School of Public Health, Kyoto University, Kyoto, Japan

Correspondence to Dr Syudo Yamasaki; yamasaki-sd@igakuken.or.jp

\section{ABSTRACT}

Objectives Women have higher rates of auditory verbal hallucinations (AVH) than men; however, less is known about sex differences in the prevalence of AVH in early, middle and late adolescence. We sought to elucidate the differences in the prevalence of AVH and to examine the degree to which these differences could be explained by differences in levels of depressive symptoms.

Design We used a cross-sectional design and a selfreported questionnaire.

Setting Participants were recruited from public junior and senior high schools in Tsu, Mie Prefecture and Kochi Prefecture, Japan.

Participants In total, 19436 students were contacted and 18250 participated. Responses from 17451 students with no missing data were analysed (aged 12-18 years, $M_{\text {age }}=15.2$ years $(S D=1.7), 50.6 \%$ girls).

Measures AVH were assessed through one of four items adopted from the schizophrenia section of the Japanese version of the Diagnostic Interview Schedule for Children. Depressive symptoms were assessed using the 12-item General Health Questionnaire.

Results The prevalence of AVH was 7.0\% among early adolescents (aged 12-13 years), 6.2\% among middle adolescents (aged 14-15 years) and 4.8\% among late adolescents (aged 16-18 years). Being female was significantly associated with a higher prevalence of AVH through adolescence $(\mathrm{OR}=1.71,95 \% \mathrm{Cl} 1.31$ to 2.23 in early adolescence; $\mathrm{OR}=1.42,95 \% \mathrm{Cl} 1.14$ to 1.76 in middle adolescence; $\mathrm{OR}=1.52,95 \% \mathrm{Cl} 1.23$ to 1.87 in late adolescence); however, these differences became non-significant after adjusting for depressive symptoms $(\mathrm{OR}=1.21,95 \% \mathrm{Cl} 0.92$ to $1.60 ; \mathrm{R}=1.00,95 \% \mathrm{Cl} 0.80$ to $1.25 ; \mathrm{OR}=1.16,95 \% \mathrm{Cl} 0.93$ to 1.44 , respectively). Conclusions Sex differences in auditory hallucinations are seen in both adult and youth populations. The higher rates of auditory verbal hallucinations seen in girls may be secondary to the differences in the rate of depressive symptoms.

\section{INTRODUCTION}

Auditory verbal hallucinations (AVH) are experienced by patients with psychotic disorders, those with non-psychotic psychiatric
Strengths and limitations of this study

- The study investigated sex differences in the prevalence of auditory verbal hallucinations in early, middle and late adolescence.

- Data were collected from a large general adolescent population, with high response rates.

- The study sample was one of the largest used for this topic.

- The study employed a cross-sectional design, hampering inferences about causal relationships based on cross-sectional associations.

disorders and a substantial part of the general population. ${ }^{1}$ Recent meta-analyses and systematic reviews reported that $5 \%-9 \%$ of the general adult population have experienced $\mathrm{AVH}^{12}$ and a median prevalence of psychotic symptoms (predominantly hearing voices) to be at $17 \%$ in a general childhood sample (aged 9-12 years) and $7.5 \%$ in adolescent samples (aged 13-18 years). ${ }^{3}$ The question on $\mathrm{AVH}$, rather than visual hallucination and persecutory thought, was very predictive of interview-verifiable psychotic symptoms and useful for screening on the general population. ${ }^{4}$

AVH often co-occur with depressive disorders among young people in clinical settings, ${ }^{5}$ and are associated with internalising problems among children in community settings. ${ }^{6}$ The presence of AVH also increases the risk of developing a wide range of psychiatric disorders and suicidal behaviour later in life. ${ }^{78} \mathrm{AVH}$ in the general population are most prevalent during adolescence, which is a critical window of vulnerability for the onset of psychosis. ${ }^{9}$

Previous studies have investigated sex differences in the prevalence of $\mathrm{AVH}$ among adolescents; however, the results are 
controversial. A meta-analysis showed that men are at a higher risk of the onset of psychotic disorders. ${ }^{10}$ However, among persons with psychosis and with clinical high-risk status, women tend to experience more auditory hallucinations than men. ${ }^{11}{ }^{12}$ In general adult populations, women have a higher incidence of positive psychotic symptoms, ${ }^{13}$ specifically auditory hallucinations, ${ }^{14}{ }^{15}$ compared with men. This sex difference may be mediated by depressive symptoms. ${ }^{13}{ }^{15}$ Similarly, girls report slightly more hallucinations than boys do. ${ }^{16}$ In contrast, Kelleher et al reported that (non-specified) psychotic symptoms are typically more prevalent among mid-adolescent boys than among girls ${ }^{17}$; however, these studies had a limited sample size. Recently, a large population-based survey of 9646 Norwegian adolescents reported that being a woman was significantly associated with a higher prevalence of $\mathrm{AVH}$, and that the difference was mediated by affective symptoms. ${ }^{18}$ However, the age range of that study sample was $16-19$ years. No study has investigated sex differences in the prevalence of AVH from early to late adolescence in a large population-based survey.

Therefore, we investigated sex differences in the prevalence of AVH among early, middle and late adolescents using data from a large population-based survey of 17451 Japanese adolescents aged 12-18 years. We also explored the degree to which any differences in this regard could be explained by differences in the levels of depressive symptoms in each age group.

\section{METHODS}

\section{Study design and procedure}

We employed an anonymous, cross-sectional survey in Japan with adolescents from public junior high schools (grades 7-9, age range $=12-15$ years) and public senior high schools (grades $10-12$, age range $=15-18$ years). The survey was conducted between 2008 and 2009, using a self-reported questionnaire. The principal investigators asked all heads and administrators of public junior high schools in Tsu, Mie Prefecture, and public junior high and senior high schools in Kochi Prefecture to participate. Of the 138 junior and 36 senior high schools invited, 47 $(34 \%)$ and $30(83 \%)$ participated, respectively.

Parents were informed of the research project by letter and asked to notify the school if they did not want their children to participate. On the day of the survey, the students were also given the choice of opting out. Each teacher reported the number of students present and absent on the day of the survey. This study was approved by the ethics committees of the Tokyo Metropolitan Institute of Medical Science, Mie University School of Medicine, and Kochi Medical School.

\section{Participants}

In total, 19436 students were recruited. Among those, 798 were absent on the day of the survey, 388 declined to participate and 18250 agreed to. Among those agreeing, 799 were excluded from the analysis because of incomplete
Table 1 Age and sex breakdown of the 17451 adolescents Total

\begin{tabular}{lllr} 
Age (years) & Male, $\mathbf{n}(\%)$ & Female, $\mathbf{n}(\%)$ & $\begin{array}{l}\text { Total } \\
\text { (n) }\end{array}$ \\
\hline $12-13$ & $1826(52.0)$ & $1687(48.0)$ & 3513 \\
\hline $14-15$ & $2956(50.3)$ & $2920(49.7)$ & 5876 \\
$16-18$ & $3838(47.6)$ & $4224(52.4)$ & 8062 \\
Total & $8620(49.4)$ & $8831(50.6)$ & 17451 \\
\hline
\end{tabular}

answers to the questions regarding psychotic-like experiences. Therefore, responses from 17451 students (aged $12-18$ years, $\mathrm{M}_{\text {age }}=15.2$ years $(\mathrm{SD}=1.7), 50.6 \%$ girls) were analysed (valid response rate $=89.8 \%$ ). Table 1 shows the adolescent groups by age and sex.

\section{Measures}

Participants were asked to complete an anonymous, self-reported questionnaire including questions about age, sex, AVH and depressive symptoms.

\section{Auditory verbal hallucinations}

AVH were assessed through one of four items adopted from the schizophrenia section of the Diagnostic Interview Schedule for Children (DISC-C). ${ }^{19}$ A Japanese version of the DISC-C was developed using a translation and back-translation method and has already been used in several previous studies conducted in Japan. ${ }^{20-24}$ AVH were assessed with the item, 'Have you ever heard voices that other people cannot hear?' Answers were provided on the following four-point scale: 'no', 'maybe', 'yes, once' and 'yes, twice or more'. In addition, we asked participants if they had experienced AVH within the last 6 months. We defined adolescents who answered 'yes, once' or 'yes, twice', and those who had experienced AVH within the last 6 months as those who had experienced AVH, and all the others as those who had not experienced AVH.

\section{Depressive symptoms}

Depressive symptoms were assessed using a 12-item General Health Questionnaire (GHQ-12). The GHQ-12 is one of the most widely used self-report measures for assessing depression. ${ }^{25}$ It has been used and validated in younger samples, as well as in adults. ${ }^{26}$ In addition, previous studies have established the validity and reliability of the Japanese version of this instrument. ${ }^{27} \mathrm{~A}$ four-point scale recorded into binary scoring (0-0-1-1) was used for the GHQ's 12 items. Responses for each question were added together to form a total score ranging from 0 (best possible) to 12 (worst possible).

\section{Statistical analysis}

We examined the effect of sex differences on the prevalence of AVH, using multivariate logistic regression. We initially tested the interaction effect between sex and age on the prevalence of $\mathrm{AVH}$, to determine if the effect of sex was modified by age. Then, we calculated the OR of the effect of sex differences on the prevalence of AVH. In addition to the crude OR, we calculated the adjusted 


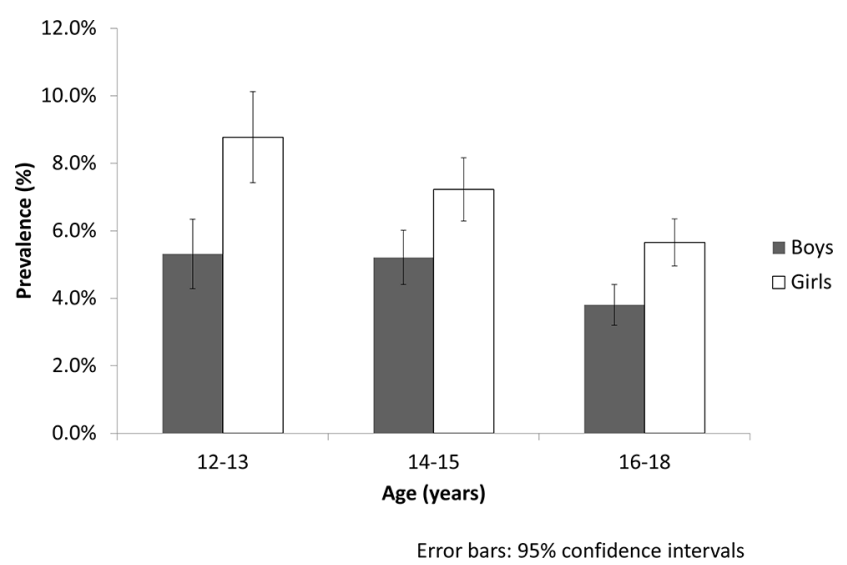

Figure 1 Prevalence of auditory hallucinations by sex and age group.

OR after adjusting for depressive symptoms. The significance level was set to $\mathrm{p}<0.05$. All statistical analyses were conducted using the IBM Statistical Package for Social Sciences (SPSS) V.21.0 for Windows (IBM, New York, USA).

\section{RESULTS}

\section{Prevalence of AVH and depressive symptoms}

The prevalence of AVH within 6 months was $5.7 \%$ among adolescents: $7.0 \%$ in early adolescence (aged 12-13 years), $6.2 \%$ in middle adolescence (aged $14-15$ years) and $4.8 \%$ in late adolescence (aged 16-18 years). The GHQ-12 scores were $2.9(\mathrm{SD}=3.0), 3.4(\mathrm{SD}=3.1)$ and 3.9 $(\mathrm{SD}=3.2)$ in early, middle and late adolescence, respectively. Adopting the validated cut-off value of $3 / 4$, the prevalence of depression was $34.3 \%$ in early adolescence, $41.5 \%$ in middle adolescence and $49.5 \%$ in late adolescence.

\section{Sex differences in the prevalence of AVH in early, middle and late adolescence}

The prevalence of AVH within the past 6 months was higher among girls than boys $\left(\chi^{2}=38.1, \mathrm{df}=1, \mathrm{p}<0.001\right)$. AVH prevalence within 6 months, by sex and age group, is shown in figure 1 . Since the interaction effect between sex and age on $\mathrm{AVH}$ prevalence was significant $(\mathrm{p}<0.001)$, we conducted logistic regression analyses on each of the three age groups. Being female was significantly associated with a higher prevalence of AVH in all three age groups (early adolescence: $\mathrm{OR}=1.71,95 \%$ CI 1.31 to 2.23 ; middle adolescence: $\mathrm{OR}=1.42,95 \% \mathrm{CI} 1.14$ to 1.76 ; late adolescence: $\mathrm{OR}=1.52,95 \%$ CI 1.23 to 1.87 ) (table 2).

\section{Sex differences in the prevalence of AVH and depressive symptoms}

After adjusting for depressive symptoms, the influence of sex differences on the prevalence of AVH did not remain significant for any of the three age groups (early adolescence: $\mathrm{OR}=1.21,95 \% \mathrm{CI} 0.92$ to 1.60 ; middle adolescence: $\mathrm{OR}=1.00,95 \%$ CI 0.80 to 1.25 ; late adolescence: $\mathrm{OR}=1.16$, $95 \%$ CI 0.93 to 1.44 ) (table 2).

\section{DISCUSSION}

This was the first study to investigate sex differences in the prevalence of AVH through early, middle and late adolescence in a large population-based survey. The results revealed that sex differences in AVH that have been observed in general adult populations are also observed among adolescents. The effect of sex was greater in early than it was in middle or late adolescence. However, for all age groups, a higher prevalence of AVH in girls may be secondary to differences in depressive symptoms.

The prevalence of AVH within the last 6 months $(5.7 \%)$ was comparable to that in previous results. ${ }^{17} 18$ Prevalence was higher in early adolescence than it was in late adolescence. This was in line with previous findings that showed that the prevalence of psychotic experiences decreases with age during adolescence. ${ }^{17}$

The prevalence of AVH was higher among female adolescents than it was among males in all three stages of adolescence. However, being female was more strongly associated with an increased prevalence of AVH in early adolescence than it was in middle to late adolescence. Moreover, the effect of being female on the prevalence of AVH did not remain after controlling for the GHQ score.

Bidirectional associations between AVH and depressive symptoms may be hypothesised. Adolescents with $\mathrm{AVH}$ may be more distressed because of their AVH; on the other hand, depressive symptoms may make adolescents more vulnerable to psychotic experiences. Adverse experiences (eg, child abuse) may be underlying the association between AVH and depression, as noted in a previous review. ${ }^{5}$ Women with psychosis have proven more likely to report abuse experiences during childhood and adolescence. ${ }^{28}$ Those with adverse experiences are more prone to internalising difficulties compared with men. ${ }^{29}$

Table 2 Logistic regression for sex differences in predicting auditory hallucination

\begin{tabular}{|c|c|c|c|c|c|c|}
\hline \multirow[b]{2}{*}{ Age (years) } & \multicolumn{3}{|c|}{ Unadjusted model } & \multicolumn{3}{|c|}{ Adjusted model* } \\
\hline & OR & $95 \% \mathrm{Cl}$ & p Value & OR & $95 \% \mathrm{Cl}$ & p Value \\
\hline $12-13$ & 1.71 & (1.31 to 2.23 ) & $<0.001$ & 1.21 & (0.92 to 1.60$)$ & 0.178 \\
\hline $16-18$ & 1.52 & (1.23 to 1.87$)$ & $<0.001$ & 1.16 & (0.93 to 1.44$)$ & 0.188 \\
\hline
\end{tabular}

*Adjusted for depressive symptoms assessed using GHQ-12 scores.

GHQ-12, 12-item General Health Questionnaire. 
Taken together, there may be a differential interaction of early trauma with biological factors (eg, genetic variations, hormonal factors) between men and women. In studies, adolescent girls were more vulnerable to hypothalamic-pituitary-adrenal axis dysregulation compared with boys ${ }^{30}$ in particular after exposure to child abuse, ${ }^{31}$ which potentially led to depression ${ }^{30}$ and psychotic symptoms at a later stage ${ }^{3233}$ Unfortunately, our cross-sectional data would not allow any further differentiation in causal relationships.

Several limitations of our study should be noted. First, information was not available about further confounding factors that may be associated with $\mathrm{AVH}$, such as socioeconomic status, family circumstances, abuse history, personality disorders or substance abuse. Second, although we had good compliance among high school students (83\%) in Kochi Prefecture, we could not achieve the same levels of participation in junior high schools in Tsu City and Kochi Prefecture, where only 47 (34\%) of the 138 junior high schools agreed to participate. This was because of refusal by the Educational Committee in Kochi Central City, which supervises most junior high schools in our targeted regions. Lastly, as noted above, we used a cross-sectional sample; therefore, we could not identify causal relationships between sex, depressive symptoms and AVH. Follow-up studies are needed to address these issues.

Despite these limitations, this study has significant strengths. First, this was one of the largest studies to date to examine the prevalence of psychotic experiences among adolescents. Second, we examined this phenomenon through three distinct stages of adolescence, enabling us to examine the effect of sex and depressive symptoms across different age ranges.

This study has some practical implications. Teachers and specialists in adolescent mental health are advised to be warier of both hallucinatory experiences and depressive symptoms among adolescents. AVH are more prevalent than earlier believed and are more frequent among girls than they are among boys. Understanding this may allow teachers and specialists to more effectively identify and help those in distress.

Acknowledgements We are grateful to all the adolescents who took part in this study, as well as the Board of Education of Tsu City and Kochi Prefecture for their cooperation and assistance in conducting this research.

Contributors YMorokuma, KE, AN, SY, SA, YMorimoto, MN, YO, TAF, SM and SS conceptualised the study. YMorokuma, KE, AN, SY, TAF and SS wrote the first draft of the manuscript. YMorokuma, KE, AN and SY conducted the statistical analyses. SY, SS and AN helped in the design of the study and the management of the database. SS and AN managed the survey process in the field. All authors contributed to and have approved the final manuscript.

Funding This study was supported by a Japan Scientific Research Grant from the Ministry of Health, Labour and Welfare (\#H19-kokoro-ippan-012); a Japan Scientific Research Grant on an Innovative Area from the Ministry of Education, Culture, Sports, Science and Technology (MEXT KAKENHI 23118002); a Japan Scientific Research Grant on an Innovative Area from the Japan Society for the Promotion of Science (JSPS KAKENHI 16H06395, 16H06398, 16K21720); a Grant-in-Aid for Challenging Exploratory Research from the Japan Society for the Promotion of Science (JSPS KAKENHI 16K13499); a Grant-in-Aid for Scientific Research (B) (KAKENHI) from the Japan Society for the Promotion of Science (JSPS KAKENHI
16H03745); and a Tokyo Metropolitan Institute of Medical Science Project Grant (Kokoronokenko H27-H31).

Competing interests None declared.

Patient consent Obtained.

Ethics approval This study was approved by the ethics committees of the Tokyo Metropolitan Institute of Medical Science, Mie University School of Medicine and Kochi Medical School.

Provenance and peer review Not commissioned; externally peer reviewed.

Data sharing statement This study was planned and conducted in accordance with the ethics committee of the Tokyo Metropolitan Institute of Medical Science. When applying to the research ethics committee for our data set, we did not request this to be released as public data. However, the data can made available to all interested researchers upon request to $\mathrm{SY}$.

Open Access This is an Open Access article distributed in accordance with the Creative Commons Attribution Non Commercial (CC BY-NC 4.0) license, which permits others to distribute, remix, adapt, build upon this work non-commercially, and license their derivative works on different terms, provided the original work is properly cited and the use is non-commercial. See: http://creativecommons.org/ licenses/by-nc/4.0/

(c) Article author(s) (or their employer(s) unless otherwise stated in the text of the article) 2017. All rights reserved. No commercial use is permitted unless otherwise expressly granted.

\section{REFERENCES}

1. van Os J, Linscott RJ, Myin-Germeys I, et al. A systematic review and meta-analysis of the psychosis continuum: evidence for a psychosis proneness-persistence-impairment model of psychotic disorder. Psychol Med 2009;39:179-95.

2. Rubio JM, Sanjuán J, Flórez-Salamanca L, et al. Examining the course of hallucinatory experiences in children and adolescents: a systematic review. Schizophr Res 2012;138:248-54.

3. Kelleher I, Connor D, Clarke MC, et al. Prevalence of psychotic symptoms in childhood and adolescence: a systematic review and meta-analysis of population-based studies. Psychol Med 2012;42:1857-63.

4. Kelleher I, Harley M, Murtagh A, et al. Are screening instruments valid for psychotic-like experiences? A validation study of screening questions for psychotic-like experiences using in-depth clinical interview. Schizophr Bull 2011;37:362-9.

5. Edelsohn GA. Hallucinations in children and adolescents: considerations in the emergency setting. Am J Psychiatry 2006;163:781-5.

6. Bartels-Velthuis AA, van de Willige G, Jenner JA, et al. Course of auditory vocal hallucinations in childhood: 5-year follow-up study. $\mathrm{Br}$ J Psychiatry 2011;199:296-302.

7. Jardri R, Bartels-Velthuis AA, Debbané $M$, et al. From phenomenology to neurophysiological understanding of hallucinations in children and adolescents. Schizophr Bull 2014;40(Suppl 4)S221-32.

8. Maijer K, Palmen SJ, Sommer IE. Children seeking help for auditory verbal hallucinations; who are they? Schizophr Res 2016.

9. Keshavan MS, Development KMS. Development, disease and degeneration in schizophrenia: a unitary pathophysiological model. $J$ Psychiatr Res 1999;33:513-21.

10. Aleman A, Kahn RS, Selten JP. Sex differences in the risk of schizophrenia: evidence from meta-analysis. Arch Gen Psychiatry 2003;60:565-71.

11. Thorup A, Albert N, Bertelsen M, et al. Gender differences in firstepisode psychosis at 5-year follow-up-two different courses of disease? Results from the OPUS study at 5-year follow-up. Eur Psychiatry 2014;29:44-51.

12. Waford RN, MacDonald A, Goines K, et al. Demographic correlates of attenuated positive psychotic symptoms. Schizophr Res 2015;166:31-6.

13. Maric N, Krabbendam L, Vollebergh W, et al. Sex differences in symptoms of psychosis in a non-selected, general population sample. Schizophr Res 2003;63:89-95.

14. Shevlin M, Murphy J, Dorahy MJ, et al. The distribution of positive psychosis-like symptoms in the population: a latent class analysis of the National Comorbidity Survey. Schizophr Res 2007;89:101-9.

15. Tien AY. Distributions of hallucinations in the population. Soc Psychiatry Psychiatr Epidemiol 1991;26:287-92. 
16. Dhossche D, Ferdinand R, Van der Ende J, et al. Diagnostic outcome of self-reported hallucinations in a community sample of adolescents. Psychol Med 2002;32:619-27.

17. Kelleher I, Keeley H, Corcoran P, et al. Clinicopathological significance of psychotic experiences in non-psychotic young people: evidence from four population-based studies. $\mathrm{Br} J$ Psychiatry 2012;201:26-32.

18. Kompus K, Løberg EM, Posserud MB, et al. Prevalence of auditory hallucinations in Norwegian adolescents: results from a populationbased study. Scand J Psychol 2015;56:391-6.

19. Costello EJ, Edelbrock CS, Costello AJ. Validity of the Nimh Diagnostic Interview Schedule for Children: a comparison between psychiatric and pediatric referrals. J Abnorm Child Psychol 1985;13:579-95.

20. Nishida A, Sasaki T, Nishimura Y, et al. Psychotic-like experiences are associated with suicidal feelings and deliberate self-harm behaviors in adolescents aged 12-15 years. Acta Psychiatr Scand 2010;121:301-7.

21. Nishida A, Shimodera S, Sasaki T, et al. Risk for suicidal problems in poor-help-seeking adolescents with psychotic-like experiences: findings from a cross-sectional survey of 16,131 adolescents. Schizophr Res 2014;159:257-62.

22. Nishida A, Tanii $\mathrm{H}$, Nishimura $\mathrm{Y}$, et al. Associations between psychotic-like experiences and mental health status and other psychopathologies among Japanese early teens. Schizophr Res 2008;99:125-33.

23. Oshima N, Nishida A, Fukushima M, et al. Psychotic-like experiences (PLEs) and mental health status in twin and singleton Japanese high school students. Early Interv Psychiatry 2010;4:206-13.

24. Watanabe N, Nishida A, Shimodera S, et al. Help-seeking behavior among Japanese school students who self-harm: results from a self-report survey of 18,104 adolescents. Neuropsychiatr Dis Treat 2012;8:561-9.

25. Goldberg DP, Rickels K, Downing R, et al. A comparison of two psychiatric screening tests. Br J Psychiatry 1976;129:61-7.

26. Kaneita Y, Ohida T, Osaki Y, et al. Association between mental health status and sleep status among adolescents in Japan: a nationwide cross-sectional survey. $J$ Clin Psychiatry 2007;68:1426-35

27. Doi Y, Minowa M. Factor structure of the 12-item General Health Questionnaire in the Japanese general adult population. Psychiatry Clin Neurosci 2003;57:379-83.

28. Fisher H, Morgan C, Dazzan P, et al. Gender differences in the association between childhood abuse and psychosis. $\mathrm{Br} J$ Psychiatry 2009;194:319-25.

29. McFadyen-Ketchum SA, Bates JE, Dodge KA, et al. Patterns of change in early childhood aggressive-disruptive behavior: gender differences in predictions from early coercive and affectionate mother-child interactions. Child Dev 1996;67:2417-33.

30. Oldehinkel AJ, Bouma EM. Sensitivity to the depressogenic effect of stress and HPA-axis reactivity in adolescence: a review of gender differences. Neurosci Biobehav Rev 2011;35:1757-70.

31. De Bellis MD, Chrousos GP, Dorn LD, et al. Hypothalamic-pituitaryadrenal axis dysregulation in sexually abused girls. J Clin Endocrinol Metab 1994;78:249-55.

32. Chaumette B, Kebir O, Mam-Lam-Fook C, et al. Salivary cortisol in early psychosis: new findings and meta-analysis. Psychoneuroendocrinology 2016;63:262-70.

33. Walker E, Mittal V, Tessner K. Stress and the hypothalamic pituitary adrenal axis in the developmental course of schizophrenia. Annu Rev Clin Psychol 2008;4:189-216. 